\title{
Comparing Psychosocial Adjustment Across the College Transition in a Matched Heterosexual and Lesbian, Gay, and Bisexual Sample
}

\author{
Alexandra Calvert Kirsch \\ Loyola University Chicago \\ Colleen S. Conley \\ Loyola University Chicago, cconley@luc.edu
}

Tracey J. Riley

tracey.riley@northwestern.edu

Follow this and additional works at: https://ecommons.luc.edu/psychology_facpubs

Part of the Psychology Commons

\section{Recommended Citation}

Kirsch, AC, C Conley, and TJ Riley. "Comparing Psychosocial Adjustment Across the College Transition in a Matched Heterosexual and Lesbian, Gay, and Bisexual Sample" in Journal of College Student Development 56(2), 2015.

This Article is brought to you for free and open access by the Faculty Publications and Other Works by Department at Loyola eCommons. It has been accepted for inclusion in Psychology: Faculty Publications and Other Works by an authorized administrator of Loyola eCommons. For more information, please contact ecommons@luc.edu. c) (i) $\Theta$

This work is licensed under a Creative Commons Attribution-Noncommercial-No Derivative Works 3.0 License. Copyright (C) 2015 American College Personnel Association (ACPA) 


\section{Comparing Psychosocial Adjustment Across the College Transition in a Matched Heterosexual and Lesbian, Gay, and Bisexual Sample}

Alexandra C. Kirsch Colleen S. Conley

We compared a matched sample of heterosexual and lesbian, gay, and bisexual (LGB) students on 5 psychosocial adjustment composites, longitudinally across the transitional first year of college. Both $L G B$ and heterosexual students experienced a significant increase in psychological distress over the first semester, along with significant decreases in psychological well-being and cognitive-affective strengths. Across the entire first year, LGB students demonstrated consistently greater psychological distress, greater cognitiveaffective vulnerabilities, and less social well-being compared to heterosexual peers. This research indicates specific challenges that $L G B$ students experience during the first year of university, suggesting opportunities for promoting successful transitions through this developmental milestone.

Research has demonstrated that lesbian, gay, and bisexual (LGB) youth and adults are at an increased risk for psychological distress and mental health symptomatology (for example, see Cochran \& Mays, 2000; King et al., 2008; McAleavey, Castonguay, \& Locke, 2011). Specifically, LGB individuals are at greater risk for depression and anxiety disorders (Biernbaum \& Ruscio, 2004; Cochran, Mays, \& Sullivan, 2003; King et al., 2008) and are more likely to engage in risk-taking behaviors including suicide attempts (Balsam, Beauchaine, Mickey, \& Rothblum, 2005; King et al., 2008), self-injurious behaviors (Balsam et al., 2005), and alcohol abuse (King et al., 2008) than heterosexual individuals.

\author{
Tracey J. Riley
}

Many psychosocial factors are important in understanding this relationship between sexual minority status and mental health adjustment. The environment and consequences associated with sexual minority status often put LGB youth and adults at an increased risk for negative and adverse life events (Oswalt \& Wyatt, 2011). Specifically, LGB individuals are much more likely to experience alienation, discrimination, victimization, and abuse as a result of their sexual minority status (Robinson \& Espelage, 2011; Williams, Connolly, Pepler, \& Craig, 2005). Sexual minority youth also typically report feeling less social support and lower satisfaction with their support networks than do heterosexual youth (Safren \& Heimberg, 1999) in both familial and peer domains (Ueno, 2005). Evidence suggests that the lack of social resources and increased experiences of discrimination that LGB individuals encounter mediate, or account for, part of the relationship between sexual minority status and mental health (Williams et al., 2005), signifying that this relationship is likely complex and multifactorial. These findings indicate the need to explore other aspects of both positive and negative adjustment and mental health and how they unfold differently for LGB and heterosexual people. Thus, in this study we examine the impact of sexual orientation not just on mental health symptomatology but also on broad psychosocial and cognitive-affective constructs, both positive and negative.

Alexandra C. Kirsch is a graduate student of Psychology; Colleen S. Conley is Associate Professor of Psychology; and Tracey J. Riley is an undergraduate student of Psychology; each at Loyola University Chicago. 
Transitioning to college is widely recognized as a stressful experience for many emerging adults (Bayram \& Bilgel, 2008; Ross, Niebling, \& Heckert, 1999), as they must master various developmental tasks including exploration, changes in relationships and roles, and new expectations and experiences that accompany this life stage (Arnett, 2006; Schulenberg, Sameroff, \& Cicchetti, 2004). Life transitions, and especially the transition to college, have been shown to increase the risk for mental health problems (Fisher \& Hood, 1987; Schulenberg et al., 2004) which might be especially challenging for LGB adolescents who are already at an increased risk for psychosocial concerns. As first-year college students are most intensely experiencing this transition and the changing relationships that accompany it, it is not surprising that the first year of college has been shown to be the most distressing (Sher, Wood, \& Gotham, 1996; Towbes \& Cohen, 1996). While the college transition is stressful for most entering students, LGB students may experience the transition and adapt differently because of their increased risk of mental health challenges and differing experiences of the college climate.

Researchers have theorized that sexual minority status may complicate the transition to college; specifically, LGB students who are struggling with their sexual identity may be less able to cope with the stressors of the college transition than their heterosexual counterparts (McAleavey et al., 2011). On the other hand, college might also provide many LGB students with their first opportunities to interact with other LGB students (Rhoads, 1997), which has been shown to reduce psychological distress (Ueno, 2005).

Despite possible benefits of the college environment, the college climate may still be more challenging for LGB students, as homophobia is still influential on many college campuses (Evans, 2002). A recent study found that a quarter of LGB students experienced harassment or violence on their campuses as a result of their sexual orientation (Rankin, Weber, Blumenfeld, \& Frazer, 2010). Additionally, Rankin and colleagues (2010) found that half of the LGB students who did not experience harassment had not come out about their sexuality due to fear of mistreatment. Some LGB students in the study who had been open with their sexual orientation in high school did not feel comfortable being out in the college environment due to a lack of social support. Given the array of challenges, it is important to understand how the transition to college may affect various aspects of psychosocial adjustment for LGB students, compared to their heterosexual peers.

A few researchers have examined sexual minority status and psychosocial adjustment in the unique college context. Debord, Wood, Sher, and Good (1998) found that LGB students and heterosexual students experienced similar decreases in symptomatology and psychological distress after the first year of university; however, other studies have demonstrated that, similar to broader samples of LGB youth, adolescents, and adults, LGB college students are at an increased risk for mental health symptomatology (Soet \& Sevig, 2006) and are more likely to feel lonelier, be more depressed, and report fewer reasons to live than heterosexual college students (Westefeld, Maples, Buford, \& Taylor, 2001). Using a national sample of college students, Oswalt and Wyatt (2011) compared LGB and heterosexual college students on a variety of mental health items, finding increased rates of anxiety, suicide attempts, help-seeking, and negative feelings and behaviors for the LGB students. Of note, this research did not thoroughly consider differential patterns of positive aspects of adjustment and functioning in LGB versus heterosexual youth. Taken 
together, these mixed results and the primary focus on negative aspects of adjustment indicate a need to further explore a broad spectrum of psychosocial adjustment during the college transition for LGB students.

In several ways this study builds on past research related to minority sexual orientation status and psychosocial adjustment during the first year of college. First, while many researchers have considered the impact of sexual orientation on general youth or adult samples, fewer have focused specifically on college students. Because past research suggests unique and potentially detrimental experiences for this population, we examined college students with a particular focus on the impact of the transition to college and the pivotal first year. Second, although research has suggested that the transition to college may be difficult for youth in general (Towbes \& Cohen, 1996), it has not directly considered how the college transition may affect LGB students as compared to their heterosexual peers. Third, much research on sexual minorities has focused on special LGB populations, such as those seeking counseling (McAleavey et al., 2011) or LGB adults of color (Meyer, 2010). Although this research is important, our study advances knowledge of LGB students by including a general college student population making an important developmental transition. Fourth, whereas previous studies have focused on limited outcomes, in this study we considered a broad array of positive and negative psychosocial factors, examining differential patterns of adjustment to college in LGB and heterosexual youth. Finally, this study builds on the longitudinal analysis of Debord and colleagues (1998) by focusing on changes in mental health and psychosocial development over time, across the first year of university, an important life transition for many emerging adults.

We examined changes in LGB and heterosexual students' psychosocial adjustment over time, starting with a precollege baseline and following students longitudinally across their first year of college. Specifically, we examined changes in five domains of psychosocial adjustment-psychological well-being, psychological distress, cognitive-affective strengths, cognitive-affective vulnerabilities, and social well-being-longitudinally across the transition to college in a matched sample of heterosexual and LGB students. Guided by past research, we utilized a theoretical framework that recognizes transitions as periods of risk for a variety of psychological, social, and behavioral changes. Additionally, in this study psychosocial adjustment is viewed as a broad concept that includes various self-perceptions, social-emotional skills, cognitive strategies, thinking styles, coping methods, internalizing symptoms, externalizing symptoms, feelings of stress, and factors related to social satisfaction and feelings of support. Further, different groups of people can experience and manage these transitions in different ways, necessitating an understanding of different trajectories. These important theoretical and methodological features were intended to broaden current understanding of adjustment to college and potential variations in how LGB and heterosexual students experience this important life transition.

\section{METHOD}

\section{Participants}

A matched sample of heterosexual $(n=46$; $M=18.39$ years of age) and LGB first-year students ( $n=46 ; M=18.39$ years of age) at a midsized urban university was selected from a larger sample $(N=1,332$; Cohort $1 n=564$; Cohort $2 n=768, M=18.5$ years of age; 71.8\% female; $73.6 \%$ White, $12.2 \%$ Asian, $6.9 \%$ Hispanic or Latino, $2.6 \%$ other race/ ethnicity, 2.3\% Black or African American, $1.5 \%$ Puerto Rican, $0.5 \%$ two or more 
TABLE 1.

Demographic Information for Matched LGB and Heterosexual Samples $(N=92)$

\begin{tabular}{|c|c|c|c|c|}
\hline & \multicolumn{2}{|c|}{ LGB Sample $(n=46)$} & \multicolumn{2}{|c|}{ Heterosexual Sample $(n=46)$} \\
\hline & Count & $\%$ & Count & $\%$ \\
\hline Age (Years) $\boldsymbol{M}(\boldsymbol{S D})$ & \multicolumn{2}{|c|}{$18.39(.33)$} & \multicolumn{2}{|c|}{$18.39(.33)$} \\
\hline \multicolumn{5}{|l|}{ Gender } \\
\hline Male & 17 & 37.0 & 17 & 37.0 \\
\hline Female & 29 & 63.0 & 29 & 63.0 \\
\hline \multicolumn{5}{|l|}{ Race/Ethnicity } \\
\hline Asian & 6 & 13.0 & 6 & 13.0 \\
\hline Black or African American & 1 & 2.2 & 1 & 2.2 \\
\hline Hispanic or Latino & 4 & 8.7 & 4 & 8.7 \\
\hline Puerto Rican & 1 & 2.2 & 1 & 2.2 \\
\hline White & 33 & 71.7 & 33 & 71.7 \\
\hline Other & 1 & 2.2 & 1 & 2.2 \\
\hline
\end{tabular}

categories, $0.4 \%$ American Indian or Alaskan) that participated in this research. Students were matched on three important identifying demographic variables: sex, age, and race/ ethnicity. Demographic characteristics for each sample can be seen in Table 1 .

\section{Procedure}

Data collected for this study came from a large-scale, multi-cohort, longitudinal research project being conducted at a single, private, Jesuit-affiliated, midsize university in an urban Midwestern setting. This larger project tracks psychosocial adjustment across the transition to university with subsequent yearly follow-ups. All entering first-year students for 2 years were recruited via e-mail to complete an online survey composed of a variety of psychosocial adjustment measures and demographic information during the week prior to enrollment (Time 1). Those who participated in the survey at Time 1 were invited via e-mail to complete subsequent rounds at the end of the first semester (Time 2) and the end of the first year (Time 3).

\section{Measures}

Demographics. With participants' consent, the university provided information on age, sex, race, and ethnicity that had been gathered as part of other institutional research. The question related to race allowed for multiple selections among the following U.S. Census Bureau categories: American Indian or Alaska Native, Asian, Black or African American, Native Hawaiian or Other Pacific Islander, or White. Students separately indicated whether they considered themselves Hispanic or Latino. Finally, incorporating citizenship information, a separate category was created to represent students who identified as Puerto Rican. Depending on the answers to these questions, race/ethnicity was recoded as American Indian or Alaska Native, Asian, Black or African American, Hispanic or Latino of any race, Native Hawaiian or Other Pacific Islander, not reported, other, Puerto Rican, two or more races, and White. Participants also self-reported their sexual orientation. The LGB group consisted of those who 
responded as lesbian, gay, or bisexual, while the heterosexual group consisted of self-reported heterosexual students.

Composite Measures of Adjustment. As psychosocial adjustment encompasses many facets of well-being, distress, cognitiveaffective styles, and social functioning, this study incorporated numerous validated measures of mental health and psychosocial adjustment that were used to create five broad composite measures: psychological well-being, psychological distress, cognitive-affective strengths, cognitive-affective vulnerabilities, social well-being. In LISREL (version 8), we performed a maximum-likelihood confirmatory factor analysis (CFA) to test this hypothesized a priori 5-factor measurement model for 21 measures used in this study using the larger sample $(N=1,332)$ from which the final sample was drawn. Following established psychometric procedures, we randomly divided the total sample in half (stratified by sex), using one half (i.e., the development sample, $n=666,31 \%$ male) to develop the measurement model, and the other half (i.e., the confirmation sample, $n=666,31 \%$ male) to confirm the cross-sample generalizability of the model. When subscales were derived from the same instrument, we allowed the unique error variances for these measures to intercorrelate in the model. Confirming the hypothesis, the proposed 5-factor model provided an acceptable goodness of fit at each time point: Time 1: $\chi^{2}(178)=4574.56$, RMSEA $=.0962$, SRMR $=.0756$, CFI $=.94$, NNFI $=.92 ;$ Time $2: \chi^{2}(178)=2973.64$, RMSEA $=.0993$, SRMR $=.0780$, CFI $=.94$, NNFI $=.93$; Time 3: $\chi^{2}(178)=2624.67$, RMSEA $=.0992$, SRMR $=.0725$, CFI $=.94$, NNFI $=.93$. Thus, we used the five CFA factors as primary dependent measures, standardizing scores on each measure across time points and averaging standardized scores to create composites. Listed below are the measures that comprise each composite and the reliability of those composites across time.

Psychological Well-Being. Psychological Well-Being was assessed at each time point $(\alpha s=.82-.84)$, with a composite of five measures: self-efficacy (17-item General Self-Efficacy Subscale; Sherer et al., 1982), self-esteem (10-item Rosenberg Self-Esteem Scale; Rosenberg, 1965), resilience (10item Connor-Davidson Resilience Scale; Campbell-Sills \& Stein, 2007), hope (8-item Adult Dispositional Hope Scale; Snyder et al., 1991), and life satisfaction (5-item Satisfaction with Life Scale; Diener, Emmons, Larsen, \& Griffin, 1985).

Psychological Distress. Psychological distress was captured at each time point $(\alpha s=.80-.87)$, by a composite of four measures: depression, anxiety, stress (7-item subscales from the Depression Anxiety Stress Scale; Lovibond \& Lovibond, 1995), and perceived stress (10-item Perceived Stress Scale; Cohen \& Williamson, 1988).

Cognitive-Affective Strengths. A cognitiveaffective strengths composite was composed of four measures, assessed at each time point ( $\alpha s=.76-.77)$ : positive thoughts (30-item Automatic Thought Questionnaire-Positive; Ingram \& Wisnicki, 1988), reappraisal (6-item subscale of Emotional Regulation Questionnaire; Gross \& John, 2003), problem-focused coping (8-item subscale of Brief COPE; Carver, 1997), and active-emotional coping (10-item subscale of Brief COPE; Carver, 1997).

Cognitive-Affective Vulnerabilities. A cognitive-affective vulnerabilities composite was composed of three measures, assessed at each time point ( $\alpha s=.79-.80)$ : negative thoughts (24-item Dysfunctional Attitudes Scale; Power et al., 1994), suppression (4item subscale of Emotional Regulation Questionnaire; Gross \& John, 2003), and avoidant coping (10-item subscale of Brief COPE; Carver, 1997). 
TABLE 2.

Results of Repeated Measures ANOVAs and Planned Contrasts Across Time

\begin{tabular}{|c|c|c|c|c|c|c|}
\hline & & $d f$ & $F$ & $\bar{p}$ & $\eta^{2}$ & Effect Size \\
\hline \multirow{9}{*}{$\begin{array}{l}\text { Psychological } \\
\text { Well-Being }\end{array}$} & Time & 2,180 & 6.63 & $.002^{* \star}$ & .069 & medium \\
\hline & Sexual Orientation & 1,90 & 1.62 & .206 & .018 & small \\
\hline & Time $\times$ Sexual Orientation & 2,180 & 0.45 & .638 & .005 & null \\
\hline & Heterosexual & & & & & \\
\hline & Time 1 vs. Time 2 & 1,45 & 11.15 & $.002 * *$ & .199 & large \\
\hline & Time 2 vs. Time 3 & 1,45 & 1.13 & .294 & .024 & small \\
\hline & LGB & & & & & \\
\hline & Time 1 vs. Time 2 & 1,45 & 5.44 & $.024 *$ & .108 & medium \\
\hline & Time 2 vs. Time 3 & 1,45 & 1.54 & .221 & .033 & small \\
\hline \multirow{9}{*}{$\begin{array}{l}\text { Psychological } \\
\text { Distress }\end{array}$} & Time & 2,180 & 3.44 & .034 & .037 & small \\
\hline & Sexual Orientation & 1,90 & 10.65 & $.002 * *$ & .106 & medium \\
\hline & Time $\times$ Sexual Orientation & 2,180 & 0.06 & .940 & .001 & null \\
\hline & Heterosexual & & & & & \\
\hline & Time 1 vs. Time 2 & 1,45 & 3.08 & .086 & .064 & medium \\
\hline & Time 2 vs. Time 3 & 1,45 & 0.28 & .601 & .006 & null \\
\hline & LGB & & & & & \\
\hline & Time 1 vs. Time 2 & 1,45 & 3.77 & .059 & .077 & medium \\
\hline & Time 2 vs. Time 3 & 1,45 & 0.54 & .467 & .012 & small \\
\hline \multirow{9}{*}{$\begin{array}{l}\text { Cognitive- } \\
\text { Affective } \\
\text { Strengths }\end{array}$} & Time & 2,180 & 8.95 & $<.001 * *$ & .090 & medium \\
\hline & Sexual Orientation & 1,90 & 0.52 & .471 & .006 & null \\
\hline & Time $\times$ Sexual Orientation & 2,180 & 1.68 & 190 & .018 & small \\
\hline & Heterosexual & & & & & \\
\hline & Time 1 vs. Time 2 & 1,45 & 13.03 & $.001 * *$ & .225 & large \\
\hline & Time 2 vs. Time 3 & 1,45 & 0.10 & .756 & .002 & null \\
\hline & LGB & & & & & \\
\hline & Time 1 vs. Time 2 & 1,45 & 7.24 & $.010 *$ & .139 & medium \\
\hline & Time 2 vs. Time 3 & 1,45 & 3.33 & .075 & .069 & medium \\
\hline \multirow{9}{*}{$\begin{array}{l}\text { Cognitive- } \\
\text { Affective } \\
\text { Vulnerabilities }\end{array}$} & Time & 2,180 & 1.31 & .272 & .014 & small \\
\hline & Sexual Orientation & 1,90 & 6.22 & $.014 *$ & .065 & medium \\
\hline & Time $\times$ Sexual Orientation & 2,180 & 1.82 & .166 & .020 & small \\
\hline & Heterosexual & & & & & \\
\hline & Time 1 vs. Time 2 & 1,45 & 6.19 & $.017 *$ & .121 & medium \\
\hline & Time 2 vs. Time 3 & 1,45 & 2.23 & .142 & .047 & small \\
\hline & LGB & & & & & \\
\hline & Time 1 vs. Time 2 & 1,45 & 0.06 & .801 & .001 & null \\
\hline & Time 2 vs. Time 3 & 1,45 & 0.00 & .986 & .000 & null \\
\hline \multirow{9}{*}{$\begin{array}{l}\text { Social } \\
\text { Well-Being }\end{array}$} & Time & 2,180 & 1.80 & .168 & .020 & small \\
\hline & Sexual Orientation & 1,90 & 3.86 & $.053+$ & .041 & small \\
\hline & Time $\times$ Sexual Orientation & 2,180 & 1.44 & .239 & .001 & null \\
\hline & Heterosexual & & & & & \\
\hline & Time 1 vs. Time 2 & 1,45 & 5.86 & $.020 *$ & .115 & medium \\
\hline & Time 2 vs. Time 3 & 1,45 & 0.27 & .605 & .006 & null \\
\hline & LGB & & & & & \\
\hline & Time 1 vs. Time 2 & 1,45 & 0.00 & .947 & .000 & null \\
\hline & Time 2 vs. Time 3 & 1,45 & 0.01 & .923 & .000 & null \\
\hline
\end{tabular}

Note. Small $\left(\eta^{2} \geq .01\right)$, medium $\left(\eta^{2} \geq .06\right)$, and large $\left(\eta^{2} \geq .14\right)$ effects (Cohen, 1988).

$* p<.05 . * * p<.01$. 
Social Well-Being. Social well-being was composed of five measures, aggregated into a composite, at each time point ( $\alpha s=.73-.77)$ : satisfaction with friends and satisfaction with parents were single-item assessments developed for this research. Participants were asked "How satisfied are you, on the whole, with the following relationships (Friends/ Parents)?" Support from friends (7 items), support from family (8 items), and general social support (8 items) were subscales of the Social Support Appraisals Scale (Vaux et al., 1986). The Cronbach's alphas for the social well-being composite were adequate at each time point ( $\left.\alpha_{s}=.73-.77\right)$.

\section{RESULTS}

\section{Comparing Heterosexual and LGB Students Over Time}

Repeated measures analyses of variance (ANOVAs) were conducted for each domain across the three time points, comparing heterosexual and LGB students as a betweensubjects factor. A priori planned contrasts

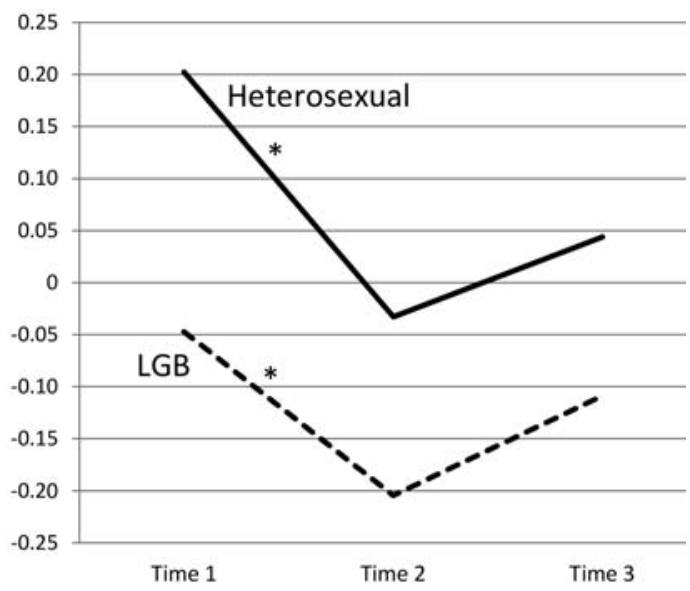

FIGURE 1. Psychological Well-Being Across the First Year of College Comparing Heterosexual and LGB Students: Significant Main Effect of Time

* Indicates a significant difference between time points. probed the significant differences across time separately for heterosexual and LGB students. In addition to statistical significance, we examined effect size (as recommended by the American Psychological Association, 2009), through $\eta^{2}$ values using Cohen's (1988) standards for small $\left(\eta^{2} \geq .01\right)$, medium $\left(\eta^{2} \geq .06\right)$, and large $\left(\eta^{2} \geq .14\right)$ effects. We noted effects that cross these thresholds and have indicated which effects are null (i.e., fall below the threshold for a small effect). Table 2 presents the results of the repeated measures ANOVAs and planned contrasts across the five domains of adjustment.

Psychological Well-Being. As displayed in Table 2, there was a medium-sized, significant main effect of time on psychological wellbeing. Although there was a small effect for sexual orientation on psychological well-being, the effect did not reach statistical significance. There was not a Sexual Orientation $\times$ Time interaction (null effect). Planned contrasts reveal a significant decrease in psychological well-being from Time 1 to Time 2 for heterosexual students (large effect) and LGB

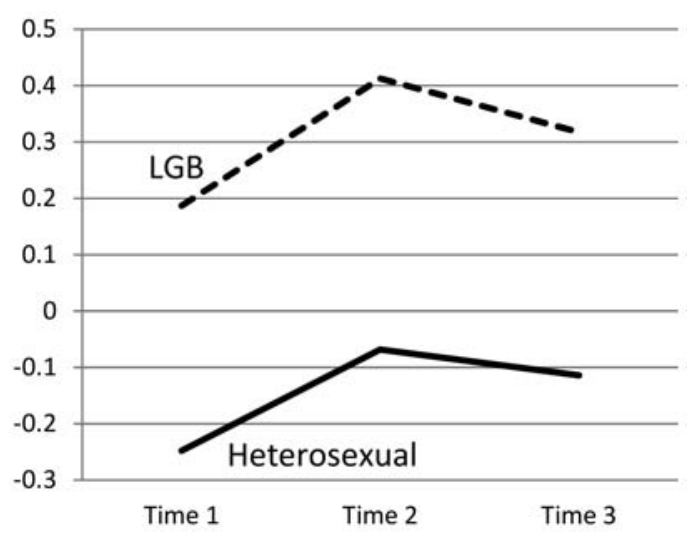

FIGURE 2. Psychological Distress Across the First Year of College Comparing Heterosexual and LGB Students:

Significant Main Effects of Time and Sexual Orientation 


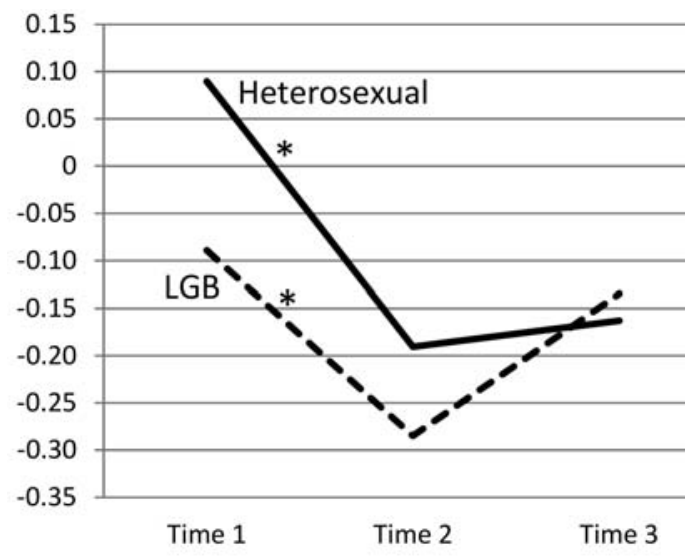

FIGURE 3. Cognitive-Affective Strengths Across the First Year of College

Comparing Heterosexual and LGB Students: Significant Main Effect of Time

* Indicates a significant difference between time points.

students (medium effect). There was not a significant change from Time 2 to Time 3 for either group (small effects).

Psychological Distress. As depicted in Table 2 and Figure 2, there were significant main effects of time (small effect), and sexual orientation (medium effect), but not a Sexual Orientation $\times$ Time interaction (null effect), on psychological distress. Planned contrasts illustrated the main effect of time: specifically, they revealed medium-sized increases in psychological distress from Time 1 to Time 2 for heterosexual and LGB students. While the overall effect of time was significant, these medium-sized effects only reached marginal significance. There was no significant change for either group from Time 2 to Time 3 (small effect for LGB students; null effect for heterosexual students). Group means in Figure 2 indicate that LGB students experienced significantly more psychological distress than did their heterosexual peers.

Cognitive-Affective Strengths. As depicted in Table 2 and Figure 3, there was a significant

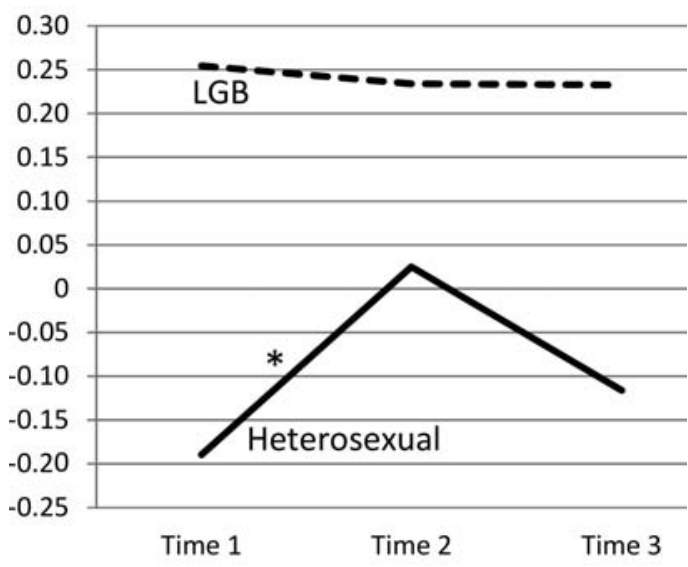

FIGURE 4. Cognitive-Affective Vulnerabilities Across the First Year of College Comparing Heterosexual and LGB Students: Significant Main Effect of Sexual Orientation

* Indicates a significant difference between time points.

main effect of time (medium effect), but not sexual orientation (null effect). The Time $x$ Sexual Orientation interaction was small but did not reach significance. Planned contrasts revealed a significant decrease in cognitiveaffective strengths from Time 1 to Time 2 for heterosexual students (large effect), and LGB students (medium effect). There was medium-sized rebound from Time 2 to Time 3 for LGB students, but this effect was only marginally significant; there was no change from Time 2 to Time 3 for heterosexual students (null effect).

Cognitive-Affective Vulnerabilities. As displayed in Table 2, there were small, but nonsignificant, effects of time and the Sexual Orientation $\times$ Time interaction on cognitiveaffective vulnerabilities. As depicted in Figure 4, there was a medium-sized significant main effect of sexual orientation, such that LGB students reported greater levels of cognitive-affective vulnerabilities across time. Planned contrasts indicated that for heterosexual students there was a medium- 


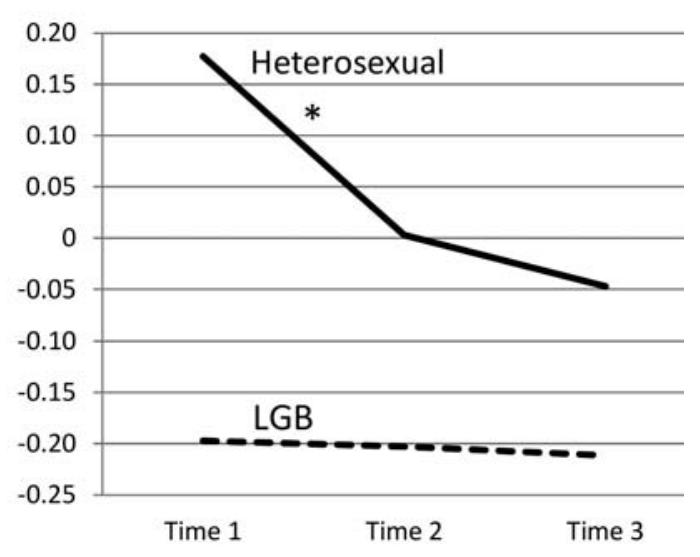

FIGURE 5. Social Well-Being Across the First Year of College Comparing Heterosexual and LGB Students: Main Effect of Sexual Orientation ${ }^{a}$

a At the border of significance; $p=.05$.

* Indicates a significant difference between time points.

sized significant increase in cognitive-affective vulnerabilities from Time 1 to Time 2 , but a small nonsignificant change between Time 2 and Time 3. For LGB individuals, there was no change in cognitive-affective vulnerabilities from Time 1 to Time 2 (null effect) nor from Time 2 to Time 3 (null effect).

Social Well-Being. As depicted in Table 2 and Figure 5, there was a small nonsignificant main effect of time and no Sexual Orientation $\times$ Time interaction (null effect). There was a small main effect for sexual orientation, such that LGB students reported poorer social wellbeing across the year than did heterosexual students, but it is important to note that this effect fell right at the border of statistical significance $(p=.05)$. Planned contrasts revealed that for heterosexual students there was a medium-sized significant decrease in social well-being from Time 1 to Time 2, but no change between Time 2 and Time 3 (null effect). For LGB students, there was no change from Time 1 to Time 2 (null effect) nor from Time 2 to Time 3 (null effect).

\section{DISCUSSION}

This study extends previous research on LGB mental health by considering both changes across the first year of college and differences between LGB and heterosexual students on five broad aspects of psychosocial adjustment. The transition to college is characterized by personal exploration, increased responsibilities, and changes in personal and social functioning (Sher et al., 1996; Towbes \& Cohen, 1996). It is important to consider the effect of this transition on LGB students, in particular, due to their increased risk of mental health symptomatology across the life span (Cochran \& Mays, 2000; King et al., 2008; McAleavey et al., 2011). This study demonstrates that for both LGB and heterosexual students there is a significant increase in psychological distress, coupled with a significant decrease in psychological well-being and cognitiveaffective strengths, over the first semester of university. Furthermore, LGB students demonstrated greater psychological distress, greater cognitive-affective vulnerabilities and less social well-being in comparison to their heterosexual peers. These main effects, combined with a lack of interactions between time and sexual orientation, suggest that LGB students and heterosexual students demonstrate similar psychosocial trajectories across time, but that LGB students demonstrate greater psychosocial challenges throughout the transitional first year.

Closer examination of Figures 4 and 5, along with the combination of main effects for sexual orientation and planned contrasts over time within groups, reveals a slightly more complex pattern: in two cases heterosexual students began with demonstrably healthier levels of functioning (in cognitive-affective vulnerabilities and social well-being), but then evidenced significant worsening over the first semester (though not steeply enough to meet 
up with their LGB peers). Main effects for sexual orientation indicate that, in contrast, LGB students began with and maintained their relatively worse levels of adjustment across the entire academic year. Thus, although the Sexual Orientation $\times$ Time interactions did not reach significance, the overall pattern of findings, including planned contrasts that differed by group over time, suggests that LGB and heterosexual students might experience different trajectories in cognitive-affective vulnerabilities and social well-being over the first year of college.

\section{Psychological Distress and Cognitive-Affective Vulnerabilities}

While previous studies have demonstrated increased mental health symptomatology in the LGB population (e.g., Cochran \& Mays, 2000), few studies have examined mental health across time or in the midst of life transitions. This study demonstrates that LGB college students also reported greater levels of psychological distress across the first year of college compared to heterosexual peers. Additionally, while both groups experienced a similar increase in psychological distress that is characteristic of the first year of college, and particularly the first semester (Bayram \& Bilgel, 2008; Towbes \& Cohen, 1996), LGB students experienced an increase on top of their already elevated levels of distress. This indicates that LGB students may be at particular risk for mental health symptomatology during the college transition, suggesting the need for services that cater specifically to this population. Further, these findings illustrate that the life stress that often accompanies major life transitions can further increase psychological distress for an already at-risk population. In this study, the transition to college had an additive effect of increasing already elevated levels of distress in LGB students.
We compared LGB and heterosexual students on a wide range of cognitive-affective strategies. Previous research has indicated that LGB adults are more likely to make use of negative cognitive-affective strategies, such as avoidant coping (Lock \& Steiner, 1999) and other unhealthy coping styles (Rosario, Schrimshaw, \& Hunter, 2009). This study indicates that LGB individuals display a tendency to engage in an even wider variety of cognitive-affective vulnerabilities, including negative thinking and emotional suppression. While there was not a significant interaction between time and sexual orientation, the significant main effect of sexual orientation, combined with a differential pattern of planned contrasts by group, revealed an important distinction: heterosexual students began with lower levels and then experienced significant increases in cognitive-affective vulnerabilities over the first semester, while LGB students demonstrated no significant changes over time, but maintained consistently greater cognitive-affective vulnerabilities over the course of the year. Perhaps LGB students' reliance on such high levels of these cognitive-affective vulnerabilities at the outset left little room for increases, even during a time of known stress. It is possible that since LGB students have had to deal with more negative life events in their youth and adolescence (Oswalt \& Wyatt, 2011), such as discrimination and victimization (Robinson \& Espelage, 2011; Williams et al., 2005), they may have developed more maladaptive methods of interpreting and handling negative events. The increased feelings of distress and cognitive-affective vulnerabilities might hint at an underlying reciprocal relationship, with greater distress contributing to LGB students' relying on negative cognitive-affective vulnerabilities; or their use of cognitiveaffective vulnerabilities may contribute to the greater experience of distress. Overall this 
study demonstrates that the college transition is a period of increased maladjustment for college students in general with LGB students at particular risk for elevated distress and cognitive-affective vulnerabilities, both at the cusp of college entry and throughout the first year. Additionally, this study indicates that interventions focused on decreasing cognitiveaffective vulnerabilities might be an important means to improve mental health functioning.

\section{Social Well-Being}

Research has indicated that sexual minorities experience less social support and satisfaction with their support networks (Safren \& Heimberg, 1999) than do heterosexual adolescents and adults. Supporting those findings, LGB students reported lower social well-being across the first year of college compared to heterosexual students in this study. While there was not a significant interaction between time and sexual orientation, planned contrasts revealed that LGB students who entered with lower social well-being experienced little change across the first year, while their heterosexual peers initially reported greater social well-being but experienced a significant decrease during the first semester. While much research has indicated that social roles and support changes across the college transition (Ross et al., 1999), this does not seem to be representative for LGB students who demonstrated a lack of social well-being throughout the transitional first year. This lack of change might be a result of combining familial, friend, and general social support and satisfaction in the social well-being composite. While heterosexual students experience a general decrease in support across domains during the transition, LGB students may experience differing relationship patterns. For example, the college transition might produce a loss of familial social support for heterosexual students, while LGB students might experience an increase in satisfaction as they are no longer living with their unsupportive families, who are often in conflict (Ueno, 2005). While these analyses focus on the broad domain of social well-being, future research might disentangle a complex pattern within specific relationship types. Additionally, this study indicates that the presumed experience of encountering greater numbers of LGB youth at college does not contribute to an increase in social well-being, as some have suggested (Rhoads, 1997; Ueno, 2005). Overall, this study indicates that although LGB students do not report worsening social well-being across the transition to college, their social well-being remains more limited than their heterosexual peers across the entire first year.

\section{Psychological Well-Being and Cognitive-Affective Strengths}

LGB youth and adolescents are at an increased risk for negative life events and mental health struggles (Oswalt \& Wyatt, 2011). However, few studies have compared LGB and heterosexual students on positive aspects of psychological well-being and cognitive-affective strengths. For many students the transition to college is accompanied by increases in stress and loss of well-being (Towbes \& Cohen, 1996). The results of this study support the understanding that the college transition is a difficult time period, as LGB and heterosexual students experienced a significant decrease in general well-being and cognitive-affective strengths over the transitional first year, with a particular dip in the first semester. Although much past research has focused on the increase in negative adjustment during the college transition (Bayram \& Bilgel, 2008; Fisher \& Hood, 1987), this study demonstrates that the first semester of college also is characterized by a loss of positive aspects of adjustmentincluding well-being and use of positive cognitive-affective strategies-regardless of 
students' sexual orientation.

The results also indicate that while LGB college students experience greater struggles in terms of psychological distress and cognitive-affective vulnerabilities, they do not demonstrate less psychological wellbeing nor fewer cognitive-affective strengths than their heterosexual counterparts. This supports previous research findings that have demonstrated few differences between LGB and heterosexual adults on more specific aspects of well-being, such as self-esteem or life satisfaction (Balsam et al., 2005). Although LGB students experience greater negative life events that can cause stress, anxiety, and depression (Oswalt \& Wyatt, 2011), these might not alter how LGB students view or understand themselves or their lives. It is intriguing that LGB students demonstrate similar patterns of well-being and cognitiveaffective strengths as heterosexual students, but concurrently demonstrate greater psychological distress and cognitive-affective vulnerabilities. Previous research has indicated that positive and negative psychosocial elements are often related to each other (Veit \& Ware, 1983; McNicholas, 2002), but our findings suggest the association might be more complex for LGB students. It is possible that LGB students can experience both well-being and distress concurrently, as they have had to adapt to environmental difficulties related to their sexual orientation while also developing resilience in the face of life stressors such as marginalization, similar to other minority populations (Nicolas et al., 2008); thus, these students might demonstrate a weaker link between distress and well-being than the heterosexual population. While most past studies have focused on negative aspects of identifying as LGB, there are positive elements of being a member of a sexual minority that might buffer against the effect of distress on general well-being and cause LGB individuals to demonstrate greater resilience to many of life's stressors (Nicolas et al., 2008; Riggle, Rostosky, Whitman, Olson, \& Strong, 2008). Future research should tease apart these unique psychosocial elements of development and attempt to identify how sexual orientation affects the associations between positive and negative adjustment and aspects associated with a minority sexual orientation that might promote positive outcomes.

\section{LIMITATIONS AND FUTURE DIRECTIONS}

Building on previous research, we assessed differences in the college transition across sexual orientation on a variety of psychosocial factors. Two key methodological strengths, using a matched sample and assessing five broad domains of psychosocial adjustment, provided a unique perspective on evaluating the firstyear transition for LGB students. Although it is important to examine the unique differences in LGB and heterosexual students, it also is crucial to take into account the intersection of racial identity with sexual orientation. The sample size and limited demographic variability left little room for examining the effect of race on either the heterosexual or LGB participants, or on the interaction between time and sexual orientation, and likely does not accurately represent the psychosocial adjustment of LGB students across all races and ethnicities. It also is important to note that the demographic groups included in this study, though derived from the U.S. Census Bureau's classifications, contain much diversity and heterogeneity within them, and many of the categories continue to be too broad to accurately represent many aspects of racial and ethnic differences. Additionally, although students were reminded of the confidential nature of their responses, some students may have withheld reporting or falsely noted their 
sexual orientation. Thus, it is possible that this study did not represent the full experience of LGB students, especially those who are not open with their sexual orientation. Future studies could compare the mental health functioning of LGB students at various stages in the coming-out process on college campuses and examine if there are stages of sexual orientation identity development that promote worse or better functioning, as has been demonstrated for other minority populations (Cross, Parham, \& Helms, 1991).

Due to the modest sample size in this study, some of the small and even medium effects failed to reach statistical significance. Future research with a larger sample is likely to evidence an even stronger pattern of findings. Additionally, the grouping of lesbian, gay, and bisexual participants into a single group does not provide the most comprehensive examination of sexual orientation status, given these distinct groups may experience the college transition in different ways. Research also could examine more specific aspects of social well-being, as LGB students may have different experiences of peer and familial social support and satisfaction. Recent U.S. Supreme Court decisions (Hollingsworth et al. v. Perry et al., 2013; United States v. Windsor, 2013), indicate that social attitudes related to the LGB population might be changing, and future research needs to examine the effect of this on psychosocial functioning. Examining this population during this evolving time period can help researchers understand the impact of changing social ideas on psychosocial functioning for minority populations. Finally, it is important to note that this research was conducted at a fairly liberal, though religiously affiliated, university where LGB students are likely to have unique experiences. The school's religious affiliation could have been a factor in participants' willingness to disclose information about sexual orientation. Although transitioning to university encompasses many of the same developmental challenges (Bayram \& Bilgel, 2008), transitioning into a religious institution as an LGB student may prompt lower feelings of acceptance compared to other institutions. Future research should examine if the current study's pattern of findings are replicated in other college settings and across larger populations.

\section{Implications and Applications}

Our findings of significant changes in psychosocial variables-specifically psychological well-being, psychological distress, and cognitive-affective strengths - across the transition to college for both LGB and heterosexual students indicate the need for additional research and increased services for entering first-year students. College administrators should provide psychoeducational resources to first-year students regarding the increased stress and mental health struggles that often characterize the first-year experience, regardless of sexual orientation. Specifically, as LGB students transition to university, they experience a significant increase in distress during the first semester compounding their already elevated experiences of distress. Further, they display consistently greater use of cognitive-affective vulnerabilities and worse social well-being across this first year. This underscores the need to provide services specifically catered to entering first-year LGB students. Programs that bring LGB students together, create a comfortable and safe campus atmosphere, and put LGB students in touch with mental health services should be provided on college campuses to best serve the needs of increasingly diverse student populations.

Correspondence concerning this article should be addressed to Alexandra C. Kirsch, Department of Psychology, Loyola University Chicago, $1032 \mathrm{~W}$. SheridanRoad, Chicago, IL 60660; akirsch@luc.edu 


\section{REFERENCES}

American Psychological Association. (2009). Publication manual of the American Psychological Association (6th ed.). Washington, DC: Author.

Arnett, J. J. (2006). The psychology of emerging adulthood: What is known, and what remains to be known? In J. J. Arnett \& J. L. Tanner (Eds.), Emerging adults in America: Coming of age in the 21st century (pp. 303-330). Washington, DC: American Psychological Association. doi:10.1037/11381-013

Balsam, K. F., Beauchaine, T. P., Mickey, R. M., \& Rothblum, E. D. (2005). Mental health of lesbian, gay, bisexual, and heterosexual siblings: Effects of gender, sexual orientation, and family. Journal of Abnormal Psychology, 114, 471-476. doi:10.1037/0021-843X.114.3.471

Bayram, N., \& Bilgel, N. (2008). The prevalence and sociodemographic correlations of depression, anxiety, and stress among a group of university students. Social Psychiatry and Psychiatric Epidemiology, 43, 667-672. doi:10.1007 /s00127-008-0345-x

Biernbaum, M., \& Ruscio, M. (2004). Differences between matched heterosexual and nonheterosexual college students on defense mechanisms and psychopathological symptoms. Journal of Homosexuality, 48(1), 125-141. doi:10.1300 /J082v48n01_06

Campbell-Sills, L. L., \& Stein, M. B. (2007). Psychometric analysis and refinement of the Connor-Davidson resilience scale (CD-RISC): Validation of a 10-item measure of resilience. Journal of Traumatic Stress, 20, 1019-1028. doi: $10.1002 /$ jts.20271

Carver, C. S. (1997). You want to measure coping but your protocol's too long: Consider the Brief COPE. International Journal of Behavioral Medicine, 4, 92-100. doi:10.1207 /s15327558ijbm0401_6

Cochran, S. D., \& Mays V. M. (2000). Lifetime prevalence of suicide symptoms and affective disorders among men reporting same-sex sexual partners: Results from NHANES III. American Journal of Public Health, 90, 573-578.

Cochran, S. D., Mays, V. M., \& Sullivan, J. G. (2003). Prevalence of mental disorders, psychological distress, and mental health services use among lesbian, gay, and bisexual adults in the United States. Journal of Consulting and Clinical Psychology, 71, 53-61. doi:10.1037/0022-006X.71.1.53

Cohen, J. (1988). Statistical power analysis for the behavioral sciences (2nd ed.). Hillsdale, NJ: Erlbaum.

Cohen, S., \& Williamson, G. (1988). Perceived stress in a probability sample of the United States. In S. Spacapan \& S. Oskamp (Eds.), The social psychology of health: Claremont symposium on applied social psychology (pp. 31-67). Newbury Park, CA: SAGE.

Cross, W. E., Jr., Parham, T. A., \& Helms, J. E. (1991). The stages of Black identity development: Nigrescence models. In R. Jones (Ed.), Black Psychology. Oakland, CA: Cobb \& Henry.

DeBord, K. A., Wood, P. K., Sher, K. J., \& Good, G. E. (1998). The relevance of sexual orientation to substance abuse and psychological distress among college students. Journal of College Student Development, 39, 157-168.

Diener, E., Emmons, R. A., Larsen, R. J., \& Griffin, S. (1985). The satisfaction with life scale. Journal of Personality Assessment, 49, 71-75. doi:10.1207/s15327752jpa4901_13
Evans, N. J. (2002). The impact of an LGBT safe zone project on campus climate. Journal of College Student Development, 43, 522-39.

Fisher, S., \& Hood, B. (1987). The stress of the transition to university: A longitudinal study of psychological disturbance, absent-mindedness, and vulnerability to homesickness. British Journal of Psychology, 78, 425-441. doi:10.1111/j.2044-8295.1987.tb02260.x

Gross, J. J., \& John, O. P. (2003). Individual differences in two emotion regulation processes: Implications for affect, relationships, and well-being. Journal of Personality and Social Psychology, 85, 348-362. doi:10.1037/0022-3514.85.2.348

Hollingsworth et al. v. Perry et al., 133 S. Ct. 2652 (2013).

Ingram, R. E., \& Wisnicki, K. S. (1988). Assessment of positive automatic cognition. Journal of Consulting and Clinical Psychology, 56, 898-902. doi:10.1037/0022-006X.56.6.898

King, M., Semlyen, J., Tai, S. S., Killaspy, H., Osborn, D., Popelyuk, D., \& Nazareth, I. (2008). A systematic review of mental disorder, suicide, and deliberate self-harm in lesbian, gay, and bisexual people. BMC Psychiatry, 8:70. doi:10.1186/1471-244X-8-70

LISREL (Version 8) [Computer software]. Skokie, IL: Scientific Software International.

Lock, J., \& Steiner, H. (1999). Gay, lesbian, and bisexual youth risks for emotional, physical, and social problems: Results from a community-based survey. Journal of the American Academy of Child \& Adolescent Psychiatry, 38, 297-304. doi:10.1097/00004583-199903000-00017

Lovibond, P. F., \& Lovibond, S. H. (1995). The structure of negative emotional states: Comparison of the Depression Anxiety Stress Scales (DASS) with the Beck Depression and Anxiety Inventories. Behavior Research and Therapy, 33, 335-343.

McAleavey, A. A., Castonguay, L. G., \& Locke, B. D. (2011). Sexual orientation minorities in college counseling: Prevalence, distress, and symptom profiles. Journal of College Counseling, 14, 127-142. doi:10.1002/j.2161-1882.2011.tb00268.x

McNicholas, S. L. (2002). Social support and positive health practices. Western Journal of Nursing Research, 24, 772-787. doi:10.1177/019394502237387

Meyer, I. H. (2010). Identity, stress, and resilience in lesbians, gay men, and bisexuals of color. Counseling Psychologist, 38, 442-454. doi:10.1177/0011000009351601

Nicolas, G., Helms, J. E., Jernigan, M. M., Sass, T., Skrzypek, A., \& DeSilva, A. M. (2008). A conceptual framework for understanding the strengths of Black youths. Journal of Black Psychology, 34, 261-280.

Oswalt, S. B., \& Wyatt, T. J. (2011). Sexual orientation and differences in mental health, stress, and academic performance in a national sample of US college students. Journal of Homosexuality, 58, 1255-1280. doi:10.1080 /00918369.2011.605738

Power, M. J., Katz, R., McGuffin, P., Duggan, C. F., Lam, D., \& Beck, A. T. (1994). The Dysfunctional Attitude Scale (DAS): A comparison of forms A and B and proposals for a new subscaled version. Journal of Research in Personality, 28, 263-276.

Rankin, S., Weber, G., Blumenfeld, W., \& Frazer, S. (2010). 2010 state of higher education for lesbian, gay, bisexual \& transgender people. Charlotte, NC: Campus Pride. 
Rhoads, R. A. (1997). A subcultural study of gay and bisexual college males: Resisting developmental inclinations. Journal of Higher Education, 68, 460-482.

Riggle, E. D. B., Rostosky S. S., Whitman, J. S., Olson, A., \& Strong, S. (2008). The positive aspects of being a lesbian or gay man. Professional Psychology: Research and Practice, 39, 210-217. doi:10.1037/0735-7028.39.2.210

Robinson, J. P., \& Espelage, D. L. (2011). Inequities in educational and psychological outcomes between LGBTQ and straight students in middle and high school. Educational Researcher, 40, 315-330. doi:10.3102/0013189X11422112

Rosario, M., Schrimshaw, E. W., \& Hunter, J. (2009). Disclosure of sexual orientation and subsequent substance use and abuse among lesbian, gay, and bisexual youths: Critical role of disclosure reactions. Psychology of Addictive Behaviors, 23, 175-184. doi:10.1037/a0014284

Rosenberg, M. (1965). Society and the adolescent self-image. Princeton, NJ: Princeton University Press.

Ross, S. E., Niebling, B. C., \& Heckert, T. M. (1999). Sources of stress among college students. College Student Journal, 33, 312-317.

Safren, S. A., \& Heimberg, R. G. (1999). Depression, hopelessness, suicidality, and related factors in sexual minority and heterosexual adolescents. Journal of Consulting \& Clinical Psychology, 67, 859-866. doi:10.1037/0022-006X.67.6.859

Schulenberg, J. E., Sameroff, A. J., \& Cicchetti, D. (2004). The transition to adulthood as a critical juncture in the course of psychopathology and mental health. Development and Psychopathology, 16, 799-806. doi:10.1017 /S0954579404040015

Sher, K. J., Wood, P. K., \& Gotham, H. J. (1996). The course of psychological distress in college: A prospective high-risk study. Journal of College Student Development, 37, 42-51.

Sherer, M., Maddux, J. E., Mercandante, E., Prentice-Dunn, S., Jacobs, B., \& Rogers, R. W. (1982). The Self-Efficacy Scale: Construction and validation. Psychological Reports, 51, 663-671. doi:10.2466/pr0.1982.51.2.663
Snyder, C. R., Harris, C., Anderson, J. R., Holleran, S. A., Irving, L. M., Sigmon, S. T., . . Harney, P. (1991). The will and the ways: Development and validation of an individualdifferences measure of hope. Journal of Personality and Social Psychology, 60, 570-585. doi:10.1037/0022-3514.60.4.570

Soet, J., \& Sevig, T. (2006). Mental health issues facing a diverse sample of college students: Results from the College Student Mental Health Survey. National Association of Student Personnel Administrators Journal, 43, 410-431.

Towbes, L. C., \& Cohen, L. H. (1996). Chronic stress in the lives of college students: Scale development and prospective prediction of distress. Journal of Youth \& Adolescence, 25, 199-217. doi:10.1007/BF01537344

Ueno, K. (2005). Sexual orientation and psychological distress in adolescence: Examining interpersonal stressors and social support processes. Social Psychology Quarterly, 68, 258-277. doi:10.1177/019027250506800305

United States v. Windsor, 133 S. Ct. 2675 (2013).

Vaux, A., Phillips, J., Holly, L., Thomson, B., Williams, D., \& Stewart, D. (1986). The Social Support Appraisals (SS-A) scale: Studies of reliability and validity. American Journal of Community Psychology, 14, 195-218. doi:10.1007 /BF00911821

Veit, C. T., \& Ware, J. E. J. (1983). The structure of psychological distress and well-being in general populations. Journal of Consulting and Clinical Psychology, 51, 730-42. doi:10.1037/0022-006X.51.5.730

Westefeld, J. S., Maples, M. R., Buford, B., \& Taylor, S. (2001). Gay, lesbian, and bisexual college students: The relationship between sexual orientation and depression, loneliness, and suicide. Journal of College Student Psychotherapy, 15(3), 71-82. doi:10.1300/J035v15n03_06

Williams, T., Connolly, J., Pepler, D., \& Craig, W. (2005). Peer victimization, social support, and psychosocial adjustment of sexual minority adolescents. Journal of Youth and Adolescence, 34, 471-482. doi:10.1007/s10964-005-7264-x 